\title{
Migrant workers play a key role in HIV-1 strain diversity in Bangladesh
}

\author{
Md. Safiullah Sarker ${ }^{1}$, Tasnim Azim¹ ${ }^{1}$ Laila N. Islam², Mustafizur Rahman ${ }^{1}$ \\ ${ }^{1}$ Virology Laboratory, Infectious Diseases Division, icddr,b, 68, Shaheed Tajuddin Ahmed Sarani, Mohakhali, Dhaka-1212, Bangladesh \\ ${ }^{2}$ Department of Biochemistry and Molecular Biology, University of Dhaka, Dhaka-1000, Bangladesh
}

\begin{abstract}
Introduction: Bangladesh is a low prevalence country for human immunodeficiency virus (HIV), and genotyping data is limited which is incomplete and mainly subtype $\mathrm{C}$ based on gag gene. In the present paper, we characterized HIV-1 strains obtained from individuals attending three HIV testing and counseling (HTC) centers from different geographic regions in Bangladesh comprehensively based on three genes.

Material and methods: A total of $40 \mathrm{HIV}$-positive blood specimens with demographic and migration history were available from individuals who attended HTC centers during 2005-2007. DNA was extracted from whole blood and sequenced using primers specific for gag, pol, and env region of HIV genome. Results: Sub-type C was the most predominant strain (45\%) followed by G-related recombinants (35\%). Other genotypes were A1 (5\%), B (2.5\%), CRF16_A2D (7.5\%), and unique recombinant form (5\%). Phylogenetic analysis indicates that the origins of these strains varied and most of them might have been imported from the Middle East on a regular basis by migrant workers.

Conclusions: This is the first study to describe comprehensive characterization of Bangladeshi HIV-1 strains. Adapted intervention programs relating to appropriate counseling and provision of information relating to risk of transmission of HIV need to aim at returnee migrant workers.
\end{abstract}

HIV AIDS Rev 2020; 19, 4: 278-283

DOI: https://doi.org/10.5114/hivar.2020.101794

Key words: developing countries, HIV-infected children, oral manifestation.

\section{Introduction}

The number of people currently living with human immunodeficiency virus (HIV)/acquired immunodeficiency syndrome (AIDS) in Bangladesh is estimated as 14,000, and that the HIV prevalence among key populations (KPs) is less than $1 \%$ [1], which is lower compared to other countries of the Asia Pacific region. However, high-risk behaviors associated with HIV, such as needle/syringe sharing, and unprotected sex are highly prevalent among KPs in Bangladesh [1]. This has already been reflected recently in a national surveillance data, which indicated a remarkable increase of HIV rate

Address for correspondence: Dr. Safiullah Sarker, MD, Virology Laboratory, International Centre for Diarrheal Diseases Research, Bangladesh (icddr,b), Mohakhali, Dhaka-1212, Bangladesh, phone: $+880-2-9827001-10$, ext. 2437 , fax: +880-2-9827075, e-mail: ssarker@icddrb.org

in people who inject drugs (PWID) from 7\% in 2011 to $27 \%$ in 2016 [1].

Existing data on genetic characterization of HIV isolates from KPs indicate that most of the strains are subtype $\mathrm{C}$, which was observed based on a partial sequence of the gag gene only [2-4]. In order to obtain better characterization of the virus, particularly for circulating recombinant forms (CRFs), at least three genetic regions - gag, pol, and env need to be considered. Here, we report the comprehensive characterization of HIV strains by sequencing three major HIV genes and provide evidence that: (i) diversity of HIV strains in the country is huge; (ii) a number of new re-

Article history:

Received: 27.03.2020

Received in revised form: 18.05.2020

Accepted: 27.05.2020

Available online: 30.12 .2020
International Journal of HIV-Related Problems

HIV \& AIDS

Re $v$ i e w 
combinant lineages were identified; (iii) most of them are imported by migrant workers especially from the Middle East.

\section{Material and methods}

\section{Sample collection}

HIV-positive blood samples were obtained from HIV testing and counseling (HTC) unit of International Centre for Diarrhoeal Disease Research, Bangladesh (icddr,b), which were collected from May, 2005 to February, 2007, and stored at $-20^{\circ} \mathrm{C}$ as described elsewhere [4]. Majority of the participants were migrant workers who returned home permanently or enjoyed a vacation.

Informed and signed consent was obtained from all study participants prior to drawing blood, and in the case of children $(n=3)$, a consent was obtained from parents/guardians. The summary of the consent paper was read out for those who could not read, and a left thumb fingerprint was obtained from those who could not sign. This study was approved by the ethical review committee of icddr,b (research protocol number: 2008-051).

\section{Sub-typing}

Pro-viral DNA was extracted from whole blood samples and was amplified by nested polymerase chain reaction (PCR) in the gag, pol, and env genes, using specific primers (Table 1). The amplified DNA was purified and sequenced on an ABI 377 automated DNA sequencer. Chromatogram sequencing files were inspected using Chromas 2.23 (Technelysium, Queensland, Australia), and consensus sequences were prepared using SeqMan II (DNAstar, Madison, WI, USA). Multiple sequence alignments were calculated using ClustalX1.81 [6]. Partial sequences of gag, pol, and env genes (at least 300 nucleotide bases) were utilized for sub-typing, using HIV genotyping tools (http://www.ncbi.nih.gov/ projects/genotyping/formpage.cgi) of the National Centre for Biotechnology Information (NCBI; National Institutes of Health, Bethesda, MD, section 2.7). All sub-types determined by genotyping tools were confirmed through a manual phylogeny, with reference strains from the Los Alamos HIV sequence database (www.hiv.lanl.gov). Sequences at the 5' and 3' ends, which could not be aligned unambiguously due to length variability of sequences were omitted from the configuration, and approximately 350 base pair sequences of $40 \mathrm{HIV}$ strains were finally analyzed. Phylogenetic analyses were conducted using a neighbor-joining method, and genetic distances were calculated with a nucleotide p-distance model in a MEGA6 software package [7]. The nucleotide sequences reported in this paper were submitted to GenBank, with MK370744-MK370841 accession number.

\section{Results}

The study population consisted of 27 males and $13 \mathrm{fe}-$ males, with ages ranging from 5 years to 75 years. The majority

Table 1. Oligonucleotide primers used in the study for PCR amplification (gag, env, and pol)

\begin{tabular}{|c|c|c|c|c|c|c|}
\hline Primer & Gene & Position (nt) & Ref. & Strand & Accession \# & Sequences (5'-3') \\
\hline 529 & gag & $884-903$ & HXB2 & Plus & K03455 & AACATMTARTRTGGGCAAGC \\
\hline 530 & gag & $764-782$ & HXB2 & Plus & K03455 & GACTAGCGGAGGCTAGAAG \\
\hline 531 & gag & $796-818$ & HXB2 & Plus & K03455 & GCGAGAGCGTCAGTATTAAGCGG \\
\hline 532 & gag & $836-857$ & HXB2 & Plus & K03455 & GGGAAAAAATTCGGTTAAGGCC \\
\hline 533 & gag & $1249-1270$ & HXB2 & Minus & K03455 & СTTCTACTACTTTTACCCATGC \\
\hline CS1 & gag & $1585-1610$ & HXB2 & Minus & K03455 & ACTATTTTATTTAATCCCAGGATTAT \\
\hline CS2 & gag & $1631-1658$ & HXB2 & Minus & K03455 & TTTGGTCCTTGTCTTATGTCCAGAATGC \\
\hline CS4 & gag & $1822-1844$ & HXB2 & Minus & K03455 & CCCTGACATGCTGTCATCATTTC \\
\hline 815 & gag & $1359-1398$ & HXB2 & Plus & K03455 & AGTGGGGGGACATCAAGCAGCCATGCAAAT \\
\hline 1114 & gag & $1631-1658$ & HXB2 & Minus & K03455 & TTTGGTCCTTGTCTTATGTCCAGAATGC \\
\hline ED5_F & env & $6517-6542$ & 06FR-CRN & Plus & EU448296 & ATGGGATCAAAGCCTAAAGCCATGTG \\
\hline ED12_R & env & $7742-7771$ & 06FR-CRN & Minus & EU448296 & AGTGCTTCCTGCTGCTCCCAAGAACCCAAG \\
\hline ED31_F & env & $6386-6414$ & Cu43 & Plus & AY586543 & CCTCAGCCATTACACAGGCCTGTCCAAAG \\
\hline 633_R & env & $7521-7538$ & HXB2 & Minus & K03455 & GGAGGGGGCATACATTGC \\
\hline 826_F & env & $6967-6998$ & HXB2 & Plus & K03455 & CGCTAGGAATTCGGCCAGTAGTATCAACTCAA \\
\hline 308_R & env & $7362-7382$ & HXB2 & Minus & K03455 & ATTACAGTAGAAAAATTCCCC \\
\hline poli5 & pol & $4177-4200$ & MN & Plus & M17449 & CACACAAAGGRATTGGAGGAAATG \\
\hline poli8 & pol & $5210-5232$ & MN & Minus & M17449 & TAGTGGGATGTGTACTTCTGAAC \\
\hline poli7 & pol & $4201-4223$ & MN & Plus & M17449 & AACAAGTAGATAAATTAGTCAGT \\
\hline poli6 & pol & $5122-5145$ & MN & Minus & M17449 & ATACATATGRTGTTTTACTAARCT \\
\hline
\end{tabular}


Table 2. Summary of HIV sub-types distribution

\begin{tabular}{|c|c|c|c|c|c|}
\hline \multirow{2}{*}{ HIV sub-type } & \multicolumn{3}{|c|}{ Country of work/Migration history } & \multirow{2}{*}{$n$} & \multirow{2}{*}{$\%$} \\
\hline & Middle East ${ }^{a}$ & India & None & & \\
\hline $\mathrm{A} 1$ & 1 & & 1 & 2 & 5.0 \\
\hline B & & & 1 & 1 & 2.5 \\
\hline $\mathrm{C}$ & 4 & 3 & 11 & $18^{\mathrm{b}}$ & 45 \\
\hline CRF02AG & 2 & & 3 & 5 & 12.5 \\
\hline CRF06cpx_A,G,J,K & 1 & & & 1 & 2.5 \\
\hline CRF09_A,G,U & 3 & & & 3 & 7.5 \\
\hline CRF13cpx_A,AE,G,J,U & 1 & & & 1 & 2.5 \\
\hline CRF16_A2D & 1 & & 2 & 3 & 7.5 \\
\hline CRF25_A,G,U & 2 & & 1 & 3 & 7.5 \\
\hline CRF43_AG & 1 & & & 1 & 2.5 \\
\hline Unique & 2 & & & 2 & 5.0 \\
\hline Total & 18 & 3 & 19 & 40 & 100 \\
\hline
\end{tabular}

${ }^{a}-$ UAE, Saudi Arabia, Bahrain, Jordan, Kuwait; ${ }^{b}$ - one HTC patient carrying sub-type C visited Saudi Arabia and India

(67.5\%) of participants were from Dhaka, followed by Sylhet (30\%) and Chattagram (2.5\%). Thirty-four (85\%) of them were heterosexual, three $(7.5 \%)$ children of HIV-positive mothers, one (2.5\%) PWID, one (2.5\%) homosexual, and one (2.5\%) with unknown risk behavior. Sub-type $\mathrm{C}$ was the most predominant strain (45\%), followed by G-related recombinant strains (35\%). Other genotypes included A1 (5\%), B (2.5\%), CRF16_A2D (7.5\%), and unique recombinant form (5\%).

The migration history is summarized in Table 2. Most of the HIV-positive individuals (18 out of 40; 45\%) travelled to the Middle East (Bahrain, Jordan, Kuwait, Saudi Arabia, and UAE), and they harbored a wide varieties of HIV strains. Among 26 migrant workers, 18 were male. All male migrant workers admitted buying sex from female sex workers while abroad. Only three (3 out of $40 ; 7.5 \%$ ) who visited India had sub-type C strains. Remaining (19 out of 40; 47.5\%) were non-migrants and spouses or sex partners of migrant workers, and majority of them (11 out of $19 ; 58 \%$ ) presented with sub-type C strains.

Three dendrograms were constructed based on gag, pol, and env regions of Bangladeshi strains along with the reference strains from different geographical regions available from the GenBank database. For better clarity of findings, only the gag tree is presented (Figure 1). Phylogenetic analysis revealed some interesting facts about the origin and transmission of the virus. First, HIV strains from our study samples dispersed all through the tree indicating extreme polyphyletic origins of local HIV strains. They clustered with HIV strains from every corner around the globe. Second, migrant workers were one of the carrier and importers of HIV strains to the country and they were likely to transmit the virus to their spouses/sex partners and children. All six spouses and partners of the returnee migrants (the Middle East) clustered together with Saudi Arabian or African strains (Figure 1, subtype A1: strain 07BD2562, CRF02_AG: strains 06BD348 and 06BD2154, CRF043_02G: strain 06BD347, sub-type C: strains
06BD2423 and 05BD952). The strain 07BD2562 (sub-type A1) was isolated from a female HTC client whose HIV-positive husband worked in the UAE and later died. We also found two strains isolated from two male children (strains 05BD1179, 05BD1230) and clustered with Indian strain (sub-type C) and Bangladeshi strain 06BD1423 whose client was a 22-year-old female. Her HIV-positive husband had travel history to India and had heterosexual contact with a sex worker there. It is presumed that the husband transferred the virus to his wife. We also identified one person with strain 07BD2718 (sub-type C) who had homosexual contact in the Saudi Arabia more likely to be infected through unprotected anal intercourse. Third, besides our male migrant workers, female migrant workers were also contributing in importing foreign HIV strains. The strain 06BD1884 (CRF16_A2D) was collected from a female who worked in Bahrain as a sex worker and is now residing in Bangladesh. It clustered with strains form a Bangladeshi couple harboring nearly identical strains 06BD2241-42 (CRF16_A2D). Another Bangladeshi strain 05BD939 (subtype C) was collected from a female sex worker from a brothel in Mumbai.

\section{Discussion}

In this study, a broad range of HIV-1 genotypes was identified suggesting multiple transmission routes for HIV epidemics in Bangladeshi population. In Bangladesh, the HIV epidemic is concentrated mainly among PWID and at some extent in female sex workers (FSW) who have been the focus for an active surveillance $[8,17]$. However, when the passively-reported cases were analyzed, another population group appeared to be vulnerable, namely migrant workers with most cases being identified through HIV testing centers; this migrants' population group who have acquired HIV while working abroad or from their spouse $[8,10]$. Approximately 

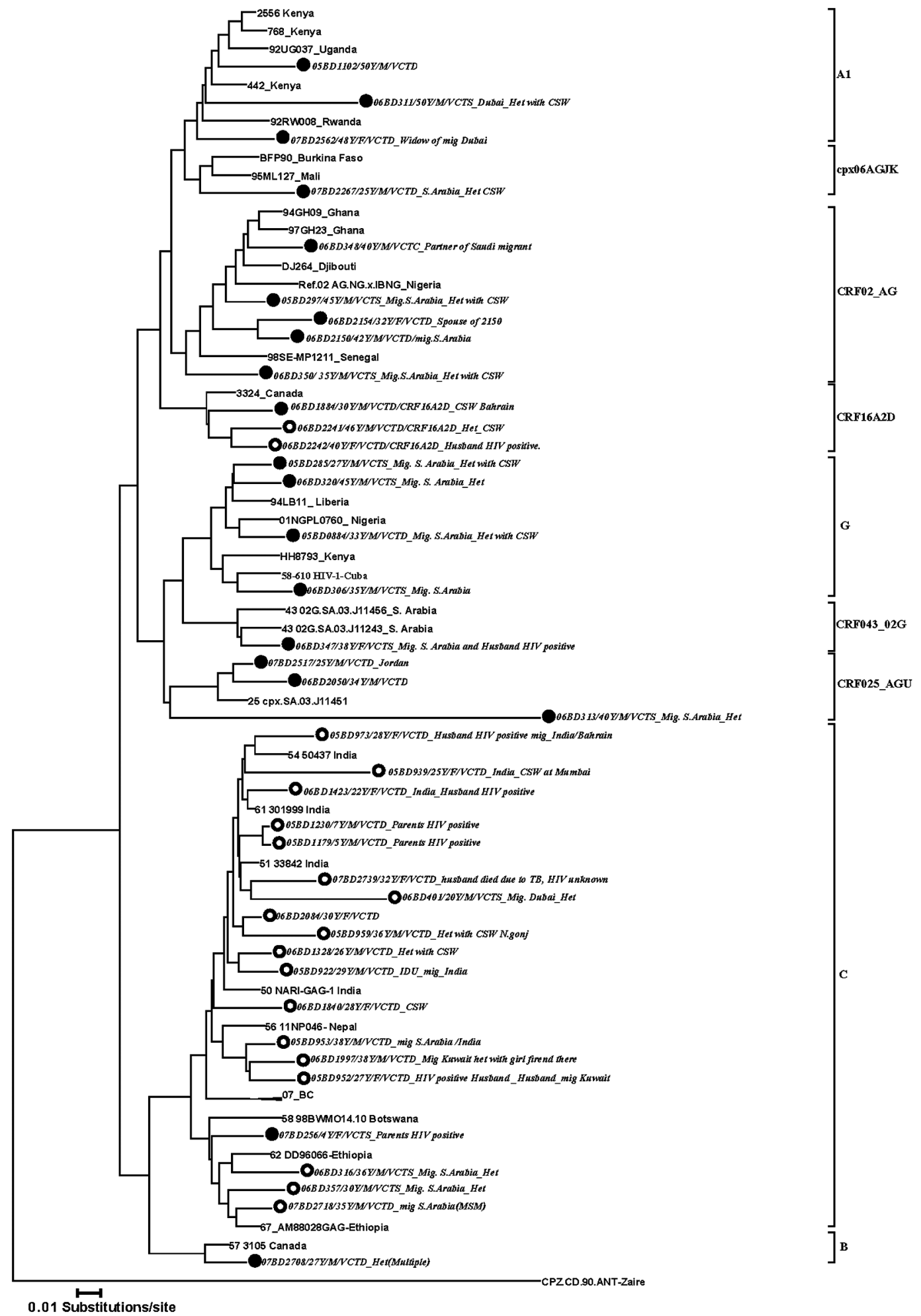

0.01 substitutions/site

Figure 1. Neighbor-joining phylogenetic tree based on nucleotide sequences of the partial gag encoding gene (350 nt bases) for HIV-1 sub-type A1, B, C, G, CRF02_AG, cpx06AGJK, CRF16A2D, CRF25_AGU, CRF043_02G. The dendrogram is rooted using simian immunodeficiency virus CPZ.CD.90.ANT. CSW - commercial sex worker, mig - migrant, Het - heterosexual transmission, VCTS - HTC unit of Sylhet, VCTD - HTC unit of Dhaka, VCTC - HTC unit of Chattagram. MSM - male having sex with male. $\mathrm{M}$ - male, $\mathrm{F}$ - female. The Bangladeshi strains are with a filled circle. Some strains published earlier are with an open circle 
$79 \%$ of people attending the icddr,b's HTC unit who were diagnosed as being infected with the HIV virus were returnee-migrants [14]. A recent study from Nepal found that a high proportion of men working abroad had sex with sex worker(s) and presented with higher rates of HIV infection than those who had not left Nepal [11].

Work-related migration is very common in Bangladesh. In a previous icddr,b study, among 1,200 married women from two districts, nearly $11 \%$ had their husband living abroad. Another study undertaken among married couples [12] showed that a large proportion of married men who travelled either within Bangladesh or abroad, reported sex with female sex workers. A few men used a condom during sex with a sex worker $(24,31 \%)$ or with their spouse $(28,31 \%)$. Although not nearly as frequent, women whose husbands were away also reported more extramarital sex compared with those women who had not spent time apart from their husbands [8]. The findings of this study was that migrants from Bangladesh was, indeed, vulnerable to HIV; however, there is still no strategy for reducing risk for migrants and their spouses without inducing a stigma.

A large number of Bangladeshi citizens are employed in the Gulf States (e.g., Saudi Arabia, Kuwait, and the United Arab Emirates). It was estimated that in Saudi Arabia, one quarter of the population are foreign nationals who make up over $50 \%$ of the work force [15], and more than $99 \%$ of them were male [16]. Before getting a resident permit, foreign nationals entering the Gulf States have to undergo HIV testing in their country of origin and if positive, they are not given a resident permit. Furthermore, migrant workers are required to renew their work permit and take a HIV test every 2 years. If anyone is found to be HIV-positive, they are deported immediately to their country of origin without counseling or treatment [13]. While abroad, these men are away from their family and likely to have unprotected sex with multiple partners, usually sex workers and occasionally, other men. Sex workers in the Gulf States are typically short-term residents with a temporary tourist visa who do not require a work permit or HIV test. Sex industry import women from nearby countries (e.g., Thailand, Myanmar, Nepal, and India) for short-term work in the Gulf States [13]. Condom use or other barrier methods are rarely used, thereby increasing their chance of infection.

One limitation of the study was using blood samples archived for more than one decade, the quality of which could be deteriorated. Therefore, we were unable to amplify $50 \%$ DNA of the stored samples, which were excluded form analysis.

\section{Conclusions}

The regular transmission of HIV by both returnee migrant workers and foreigners is expected to increase the prevalence and diversity of HIV among the Bangladeshi population. Adapted intervention programs, such as counseling and provision of information relating to risk of contracting HIV, need to aim at migrant workers at different points of travelling: when leaving home-country, while working overseas, upon their return to home-country, and while staying at home-country. In conclusion, the study revealed a broad genetic diversity of HIV-1 strains in Bangladeshi population, which was mainly caused by returnee migrant workers. This finding warrants the inclusion of migrant workers as a key population for HIV in Bangladesh.

\section{Acknowledgement}

icddr,b acknowledges with gratitude the commitment of Marie Stopes, Bangladesh to its research effort. icddr,b also gratefully acknowledges the following donors who provided unrestricted support: the Government of the People's Republic of Bangladesh, the Global Affairs Canada (GAC), the Swedish International Development Cooperation Agency (Sida), and the Department for International Development (UKAid).

This research protocol was funded by icddr,b's core donors. Also, VCT activity (ACT-00726) was funded by the Marie Stopes, Bangladesh, grant number GR-01236. The funders did not participate in the study design, data collection, and analysis as well as decision to publish or preparation of the manuscript.

\section{Conflict of interest}

The authors declare no conflict of interest with respect to the research, authorship, and/or publication of this article.

\section{References}

1. NASP. Behavioural and serological surveillance amongst key populations at risk of HIV in selected areas of Bangladesh, 2016. National AIDS/STD Program, Directorate General of Health Services, Ministry of Health and Family Welfare, Government of Bangladesh, 2016.

2. Azim T, Bogaerts J, Yirrell DL, et al. Injecting drug users in Bangladesh: prevalence of syphilis, hepatitis, HIV and HIV subtypes. AIDS 2002; 16: 121-123.

3. Ljungberg K, Hassan MS, Islam MN, et al. Subtypes A, C, G, and recombinant HIV type 1 are circulating in Bangladesh. AIDS Res Hum Retroviruses 2002; 18: 667-670.

4. Sarker MS, Rahman M, Yirrell D, et al. Molecular evidence for polyphyletic origin of human immunodeficiency virus type 1 subtype C in Bangladesh. Virus Res 2008; 135: 89-94.

5. Tovanabutra S, Watanaveeradej V, Viputtikul K, et al. A new circulating recombinant form, CRF15_01B, reinforces the linkage between IDU and heterosexual epidemics in Thailand. AIDS Res Hum Retroviruses 2003; 19: 561-567.

6. Thompson JD, Higgins DG, Gibson TJ. CLUSTAL W: improving the sensitivity of progressive multiple sequence alignment through sequence weighting, position-specific gap penalties and weight matrix choice. Nucleic Acids Res 1994; 22: 4673-4680.

7. Tamura K, Stecher G, Peterson D, Filipski A, Kumar S. MEGA6: molecular evolutionary genetics analysis version 6.0. Mol Biol Evol 2013; 30: 2725-2729.

8. Azim T, Khan SI, Haseen F, et al. HIV and AIDS in Bangladesh. J Health Popul Nutr 2008; 26: 311.

9. Badreddine S, Smith K, Van Zyl H, et al. Identification and characterization of HIV type 1 subtypes present in the Kingdom of Saudi Arabia: high level of genetic diversity found. AIDS Res Hum Retroviruses 2007; 23: 667-674.

10. Urmi AZ, Leung DT, Wilkinson V, Miah MA, Rahman M, Azim T. Profile of an HIV testing and counseling unit in Bangladesh: majority of new diagnoses among returning migrant workers and spouses. PLoS One 2015; 10: e0141483. 
11. Nepal B. Population mobility and spread of HIV across the IndoNepal border. J Health Popul Nutr 2007; 25: 267.

12. Mercer A, Khanam R, Gurley E, Azim T. Sexual risk behavior of married men and women in Bangladesh associated with husbands' work migration and living apart. Sex Transm Dis 2007; 34: 265-273.

13. Shah SA, Khan OA, Kristensen S, Vermund SH. HIV-infected workers deported from the Gulf States: impact on Southern Pakistan. Int J STD AIDS 1999; 10: 812-814.

14. Shahrin L, Leung DT, Matin N, Kawser CA, Pervez MM, Chisti MJ. Clinical profile of hospitalized HIV-infected children in Bangladesh, a low-HIV-prevalence country. Paediatrics and International Child Health 2014; 34: 133-137.

15. Kandela P. Gulf states test foreigners for AIDS. BMJ 1994; 308: 617.

16. Barkat A, Ahsan M. Gender and migration from Bangladesh. International Labour Organization 2014.

17. Azim T, Khan SI, Nahar Q, et al. 20 years of HIV in Bangladesh: experiences and way forward. World Bank 2009. 